\title{
Basics of changes in hemodynamic monitoring in sepsis care
}

\author{
Jae Chol Yoon ${ }^{1}$, Won Young Kim ${ }^{2}$ \\ ${ }^{1}$ Department of Emergency Medicine, Research Institute of Clinical Medicine of Chonbuk National University and Biomedical Research Institute \\ of Chonbuk National University Hospital, Jeonju-si, Republic of Korea; ${ }^{2}$ Department of Emergency Medicine, University of Ulsan College of \\ Medicine, Asan Medical Center, Seoul, Republic of Korea \\ Correspondence to: Won Young Kim. Department of Emergency Medicine, University of Ulsan College of Medicine, Asan Medical Center, 88, \\ Olympic-ro 43-gil, Songpa-gu, Seoul 05505, Korea. Email: wonpia73@naver.com. \\ Provenance: This is an invited article commissioned by the Section Editor Xue-Zhong Xing [National Cancer Center (NCC)/Cancer Hospital, \\ Chinese Academy of Medical Sciences (CAMS) and Peking Union Medical College (PUMC), Beijing, China]. \\ Comment on: Hernández G, Ospina-Tascón GA, Damiani LP, et al. Effect of a resuscitation strategy targeting peripheral perfusion status vs serum \\ lactate levels on 28-day mortality among patients with septic shock: the ANDROMEDA-SHOCK randomized clinical trial. JAMA 2019;321:654-64.
}

Submitted Jul 05, 2019. Accepted for publication Sep 01, 2019.

doi: $10.21037 /$ jtd.2019.09.43

View this article at: http://dx.doi.org/10.21037/jtd.2019.09.43

Sepsis is a life-threatening organ dysfunction caused by a dysregulated host response to an infection, and septic shock is a subset of sepsis in which profound circulatory, cellular, and metabolic abnormalities occur, associated with a greater risk of mortality compared to sepsis alone (1). Septic shock should be considered a medical emergency, and focus must be placed on timely intervention, including early identification and treatment of the infection through appropriate antimicrobial therapy, source control when applicable, and reversal of hemodynamic instability through fluid resuscitation and vasopressor use $(2,3)$. However, intravenous fluids and vasopressors could harm the patient. Therefore, these therapies must be adjusted in response to markers of resuscitation adequacy. In agreement with these reports, the Surviving Sepsis Campaign (SSC) 2016 Guidelines have removed the standard goals, such as of central venous pressure (CVP) and central venous oxygen saturation (4). Removing the most specific endpoints emphasize on the importance of serum lactate levels in patient-specific customization of the hemodynamic therapy and suggest performing hemodynamic resuscitation by repeated measurement of the blood lactate levels every 2 to 4 hours until normalization (weak recommendation, low quality of evidence) (4,5). Moreover, the Third International Consensus Definitions for Sepsis and Septic Shock (Sepsis-3) has included hyperlactatemia over $2 \mathrm{mmol} / \mathrm{L}$ in the revised definition of septic shock $(1,6,7)$.

While an elevated serum lactate level suggests tissue hypoperfusion (8), and lactate-guided resuscitation in early sepsis showed non-inferiority in a randomized controlled study (9), it can be elevated in conditions other than tissue hypoperfusion, including increased adrenergicdriven aerobic glycolysis and impaired lactate removal $(5,10)$. In other words, there is a risk of over-resuscitation if hyperlactatemia in sepsis is associated with lactate production or clearance rather than with hypoperfusion. Moreover, lactate clearance is relatively slow in providing timely feedback to clinicians regarding the treatment outcome (11), and serum lactate measurements may not be universally available, especially in the developing countries. Therefore, investigating the alternative resuscitation targets is an important research priority in sepsis.

Regarding the best resuscitation end point to assess adequate tissue perfusion, the ANDROMEDA-SHOCK randomized clinical trial in a recent issue of $\mathcal{F} A M A$, in which 424 adults with septic shock were randomized to peripheral perfusion-guided resuscitation using capillary refill time (CRT) and lactate-guided resuscitation, postulated a potential alternative to assess adequate tissue perfusion in patients with early septic shock (12). The intervention period was 8 hours after randomization. Lactate-guided resuscitation represented the current guidelines and was intended to titrating resuscitation based on the lactate level measured every 2 hours, with a target of greater than $20 \%$ reduction of lactate every 2 hours or normalization of the lactate level. CRT-guided resuscitation was guided by 
CRT measured every 30 minutes with a target of less than 3 seconds of CRT. The enrolled patients had typical features of septic shock. Most patients (71\%) were admitted from the emergency department, and the common infections were pneumonia and intra-abdominal infections. The resuscitation protocol was a well-operable and stepwise approach (step 1: fluid responsiveness; step 2: vasopressor test; step 3: inodilator test) and could be applicable widely. This protocol is meaningful in the view of using dynamic variables to assess fluid responsiveness and guide additional fluid therapy rather than a static variable, such as CVP.

The primary outcome was 28 -day all-cause mortality. The study showed $8.5 \%$ reduction (approximately $20 \%$ of relative risk reduction) of mortality in the CRT-guided resuscitation group (34.9\% in the CRT group vs. $43.4 \%$ in the lactate group, $\mathrm{P}=0.06)$, but without statistical significance. The authors concluded that a resuscitation strategy targeting normalization of CRT, compared to a strategy targeting serum lactate levels, did not reduce the 28-day all-cause mortality. Although this conclusion suggests a negative trial, there is a concern regarding the sample size calculation that this result could be significant statistically if the sample size or magnitude of effect was increased. The sample size of 420 patients was calculated with $90 \%$ power to detect an absolute reduction rate of $15 \%$ for lack of previous data to calculate the power with CRT. Although the authors' assumption that $15 \%$ was the smallest clinically important difference in 28-day mortality between CRT and lactate-guided group made it a feasible sample size, this raises an issue of underpowering to answer the study question. The ANDROMEDA-SHOCK trial is not a non-inferiority study concluding that CRT-guided resuscitation is no worse than lactate-guided resuscitation, but there was less organ dysfunction at 72 hours in the CRT-guided group, as evidenced by mean 72-hour sequential organ failure assessment (SOFA) scores of $5.6 \mathrm{vs}$. 6.6 [95\% confidence interval (CI), -1.97 to $-0.02 ; \mathrm{P}=0.045]$. CRT-guided resuscitation is unlikely to worsen the outcome clinically. In other words, CRT-guided resuscitation is not inferior to lactate-guided resuscitation.

The resuscitation measures were similar in both groups, except for the resuscitation volume within the first 8 hours [mean difference $-408(-705$ to -100$) \mathrm{mL}$ ]. This result addressed that major therapies such as fluid administration and vasopressor use might be similar, regardless of the monitoring method. However, differences in the resuscitation volume within the first 8 hours might be attributed to the frequency of reassessment and persistent hyperlactatemia. The trend of reduction in mortality in CRT-guided resuscitation was presumed to be for the same reason. CRT was assessed every 30 minutes and lactate every 2 hours. Skin perfusion is a flow-sensitive variable, so it might respond rapidly following fluid loading in fluid responsive patients. The study on evolution of perfusion parameters during septic shock resuscitation demonstrated that CRT showed the earliest normalization, and the normal value of CRT (less than 3 seconds) at 6 hours was independently associated with successful resuscitation, defined as normalization of the lactate level (13). Other studies including a cohort of surviving septic shock patients confirmed that the normality of CRT increased from $46 \%$ to $70 \%$ in the patients after 2 hours of fluid resuscitation, while the lactate level was normalized in only $52 \%$ of the patients at 24 hours (14). This study could not be completely blinded to the treating physician. Therefore, the treating physician might have used both laboratory and physiologic parameters in the intervention period. It can be presumed that frequent reassessment in CRT-guided resuscitation and rapid response of CRT might result in a more meticulous resuscitation compared to lactate-guided resuscitation. Slow normalization of lactate following fluid resuscitation and hyperlactatemia from mechanisms other than tissue hypoperfusion could be presumed to cause over-resuscitation in the early phase. The SSC guideline also recommends frequent reassessment of the hemodynamic status to guide the additional fluid therapy following the initial fluid resuscitation (4).

CRT could be used as a target for fluid resuscitation in septic shock because of the simplicity of its assessment, bedside applicability, rapid recovery following fluid resuscitation, and availability in a resource-limited setting. A CRT $>3.5 \mathrm{~s}$ indicates poor peripheral perfusion and, if associated with hyperlactatemia, marked circulatory failure (15). However, there are several limitations of CRT. CRT measurement should be standardized, including the application site, pressure time and method, and normal value. In this study, authors standardized CRT assessment by applying a microscope slide to the finger pulp for 10 seconds of blanching and counting the time to reperfusion using a chronometer. Quantitative CRT measured using a modified pulse oximeter device can predict hyperlactatemia and was suggested as an objective method to assess CRT (16). Pressing the nail bed with 3-7 N (3 N is strength needed to lift an object weighing $300 \mathrm{~g}$ ) for 2 seconds was suggested as the optimal pressing strength and time for measuring CRT (17). Although authors claimed that training and using a chronometer could reduce interrater variability, this 
problem should be considered (18).

In summary, the ANDROMEDA-SHOCK trial showed that a resuscitation strategy targeting normalization of CRT, compared to a strategy targeting serum lactate levels, did not reduce the 28-day all-cause mortality; however, CRT assessment can be conducted in a simple, inexpensive, and standardized way and may play a role in the titration of fluids and vasopressors during early resuscitation in septic shock, especially in resource-limited settings. This study might postulate the importance of basic care, clinical examination, and frequent reassessment in the early resuscitation phase. Future studies should be continued on the usefulness of the physiologic parameters, including CRT as microcirculatory resuscitation end points, in various shock states.

\section{Acknowledgments}

None.

\section{Footnote}

Conflicts of Interest: The authors have no conflicts of interest to declare.

Ethical Statement: The authors are accountable for all aspects of the work in ensuring that questions related to the accuracy or integrity of any part of the work are appropriately investigated and resolved.

\section{References}

1. Singer M, Deutschman CS, Seymour CW, et al. The third international consensus definitions for sepsis and septic shock (sepsis-3). JAMA 2016;315:801-10.

2. Yoon JC, Kim WY. Progression in optimal sepsis care: the sooner, the better? J Emerg Crit Care Med 2017;1:14.

3. Shin TG, Kim YJ, Ryoo SM, et al. Early vitamin C and thiamine administration to patients with septic shock in emergency departments: propensity score-based analysis of a before-and-after cohort study. J Clin Med 2019. doi: $10.3390 / \mathrm{jcm} 8010102$.

4. Rhodes A, Evans LE, Alhazzani W, et al. Surviving sepsis campaign: international guidelines for management of sepsis and septic shock: 2016. Crit Care Med 2017;45:486-552.

5. Ryoo SM, Ahn R, Lee J, et al. Timing of repeated lactate measurement in patients with septic shock at the emergency department. Am J Med Sci 2018;356:97-102.

6. Ko BS, Kim K, Choi SH, et al. Prognosis of patients excluded by the definition of septic shock based on their lactate levels after initial fluid resuscitation: a prospective multi-center observational study. Crit Care 2018;22:47.

7. Ryoo SM, Kang GH, Shin TG, et al. Clinical outcome comparison of patients with septic shock defined by the new sepsis-3 criteria and by previous criteria. J Thorac Dis 2018;10:845-53.

8. Ryoo SM, Kim WY. Clinical applications of lactate testing in patients with sepsis and septic shock. J Emerg Crit Care Med 2018;2:14.

9. Jones AE, Shapiro NI, Trzeciak S, et al. Lactate clearance vs central venous oxygen saturation as goals of early sepsis therapy: a randomized clinical trial. JAMA 2010;303:739-46.

10. Ryoo SM, Lee J, Lee YS, et al. Lactate level versus lactate clearance for predicting mortality in patients with septic shock defined by sepsis-3. Crit Care Med 2018;46:e489-95.

11. Angus DC. How best to resuscitate patients with septic shock? JAMA 2019;321:647-8.

12. Hernández G, Ospina-Tascón GA, Damiani LP, et al. Effect of a resuscitation strategy targeting peripheral perfusion status vs serum lactate levels on 28-day mortality among patients with septic shock: the ANDROMEDA-SHOCK randomized clinical trial. JAMA 2019;321:654-64.

13. Hernandez G, Pedreros C, Veas E, et al. Evolution of peripheral vs metabolic perfusion parameters during septic shock resuscitation. A clinical-physiologic study. J Crit Care 2012;27:283-8.

14. Hernandez G, Luengo C, Bruhn A, et al. When to stop septic shock resuscitation: clues from a dynamic perfusion monitoring. Ann Intensive Care 2014;4:30.

15. Alegría L, Vera M, Dreyse J, et al. A hypoperfusion context may aid to interpret hyperlactatemia in sepsis-3 septic shock patients: a proof-of-concept study. Ann Intensive Care 2017;7:29.

16. Morimura N, Takahashi K, Doi T, et al. A pilot study of quantitative capillary refill time to identify high blood lactate levels in critically ill patients. Emerg Med J 2015;32:444-8.

17. Kawaguchi R, Nakada TA, Oshima T, et al. Optimal pressing strength and time for capillary refilling time. Crit Care 2019;23:4.

18. Brabrand M, Hosbond S, Folkestad L. Capillary refill time: a study of interobserver reliability among nurses and nurse assistants. Eur J Emerg Med 2011;18:46-9.

Cite this article as: Yoon JC, Kim WY. Basics of changes in hemodynamic monitoring in sepsis care. J Thorac Dis 2019;11(10):E168-E170. doi: 10.21037/jtd.2019.09.43 